\title{
Mathematical analysis of complex SIR model with coinfection and density dependence
}

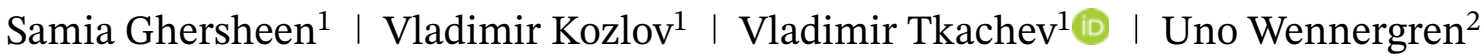

${ }^{1}$ Department of Mathematics, Linköping University, Linköping, Sweden

${ }^{2}$ Department of Physics, Chemistry, and Biology, Linköping University, Linköping, Sweden

\section{Correspondence}

Vladimir Tkachev, Department of Mathematics, Linköping University, 58183 Linköping, Sweden.

Email: vladimir.tkatjev@liu.se
An SIR model with the coinfection of the two infectious agents in a single host population is considered. The model includes the environmental carry capacity in each class of population. A special case of this model is analyzed, and several threshold conditions are obtained, which describes the establishment of diseases in the population. We prove that, for small carrying capacity $K$, there exists a globally stable disease-free equilibrium point. Furthermore, we establish the continuity of the transition dynamics of the stable equilibrium point, that is, we prove that, (1) for small values of $K$, there exists a unique globally stable equilibrium point, and (b) it moves continuously as $K$ is growing (while its face type may change). This indicates that the carrying capacity is the crucial parameter and an increase in resources in terms of carrying capacity promotes the risk of infection.

\section{KEYWORDS}

carrying capacity, coinfection, global stability, SIR model

\section{1 | INTRODUCTION}

Coinfection means that a person is affected by more than one infectious agent at a time. Many pathogens that infect humans (eg, viruses, bacteria, protozoa, fungal parasites) often coexist within individuals. ${ }^{1}$ Consequently, coinfection of individual hosts by multiple infectious agents is a phenomenon that is very frequently observed in natural populations. The study of complete dynamics of this phenomenon involves many complexities. By understanding the multiple interactions that cause coinfection, we will be able to understand and intelligently predict how a suite of coinfections will together respond to medical interventions as well as other environmental changes. ${ }^{2}$

Mathematical analysis of infectious diseases has significant importance in infectious disease epidemiology to study not only the dynamics of disease, but it is also very helpful to design the practical controlling strategies. Mathematical analysis and models have successfully explained previously mystifying observations and played a central part in public health strategies in many countries. ${ }^{3,4}$

Many mathematical studies exist on interaction of multiple-strain and multiple-disease cointeractions. ${ }^{5-13}$ Some of the studies are about the general dynamics of coinfection. ${ }^{14-16}$

Because coinfection includes a lots of complexities and therefore a lot of different classes and interactions between classes. This implies the necessity of advanced mathematical analysis to handle this problem. However, there is also a risk that one reaches the boarder of what is actually possible to analyze. Previously, Allen et $\mathrm{al}^{17}$ studied an SI model with density-dependent mortality and coinfection in a single host where one strain is vertical transmitted and the other is horizontally transmitted and the model has application on hantavirus and arenavirus, and Gao et al ${ }^{18}$ studied an 
SIS model with dual infection. Simultaneous transmission of infection and no immunity has been considered. The study revealed that the coexistence of multiple agents caused coinfection and made the disease dynamics more complicated. It was observed that coexistence of two disease can only occur in the presence of coinfection. In the above models, they considered that the number of births per unit time is constant. Our approach in this paper is to show different degrees of complexities and to suggest a model that is an extension of the model studied by Ghersheen et al, ${ }^{19}$ where an SIR model was analyzed. That model describes the coinfection of the two infectious agents in a single host population with an addition of limited growth of susceptible in terms of carrying capacity. Previously, to diminish the complexity, it was considered that there is no interaction between two single infectious agents and that coinfection only occurs as a result of interaction between coinfected class and single infected class, and coinfected class and susceptible class. In this model, we add more complexity by relaxing all these assumptions and adding the density dependence in each class. The addition of two viruses with density dependence for human population is a new modeling perspective because death and birth rates change over time in human population. One billion out of eight billions of human population is facing the problem of hunger due to the lack of resources that can fluctuate the birth rates over time. Therefore, in contrast to the works of Allen et $\mathrm{l}^{17}$ and Gao et al ${ }^{18}$ we first analyze the model with the same interaction terms, but only the growth of the susceptible class is limited in terms of carrying capacity, because an infected and recovered population is regulated by its death rate. We assume that infected and recovered individuals cannot reproduce.

We formulate an SIR model with coinfection and logistic-type population growth in each class population to study the effects of carrying capacity on disease dynamics. However, contrary to the work of Allen et al, ${ }^{17}$ to study the global behavior of the system, the reduction of the system is needed to some sense. Therefore, in the first place, we consider the relative simplified model and only limit the growth of susceptible populations in terms of carrying capacity. We assume that the infected population cannot reproduce due to infection. We carried out the local and global stability analysis using a generalized Volterra function for each stable point to study the complete dynamics of disease. We analyze an SIR model with complete cross immunity. In Section 2, we begin with the description of a full model, and in Section 2, we presented and analyzed a submodel. In Section 3, we recall some general facts about the SIR model (1), and in the remaining sections, we characterize all equilibrium points and give the results regarding local and global stability.

\section{2 | FORMULATION OF THE MODEL}

The first model is the relevant extension to the model presented in the work of Ghersheen et al ${ }^{19}$ to understand the complex dynamics of coinfection. Firstly, we assume that a susceptible individual can be infected with either one or both infectious agents as a result of contact with coinfected individual. Secondly, coinfection occurs as a result of contact between two single infected individuals or between a coinfected person and a single infected person. This process is illustrated in the compartmental diagram in Figure 1.

Following other works, ${ }^{17,19-21}$ we assume limited population growth by making the per capita reproduction rate depend on the density of population. We also consider the recovery of each infected class. The SIR model is then described by the

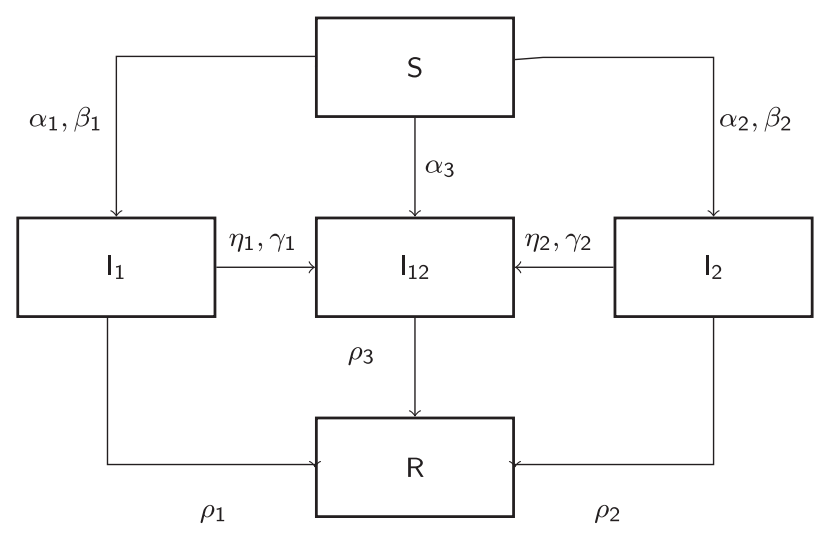

FIGURE 1 Flow diagram for the two-strains coinfection model 
system of five ordinary differential equations as follows:

$$
\begin{aligned}
S^{\prime} & =\left(b_{1}\left(1-\frac{N}{K_{1}}\right)-\alpha_{1} I_{1}-\alpha_{2} I_{2}-\left(\beta_{1}+\beta_{2}+\alpha_{3}\right) I_{12}-\mu_{0}\right) S, \\
I_{1}^{\prime} & =\left(b_{2}\left(1-\frac{N}{K_{2}}\right)+\alpha_{1} S-\eta_{1} I_{12}-\gamma_{1} I_{2}-\mu_{1}\right) I_{1}+\beta_{1} S I_{12}, \\
I_{2}^{\prime} & =\left(b_{3}\left(1-\frac{N}{K_{3}}\right)+\alpha_{2} S-\eta_{2} I_{12}-\gamma_{2} I_{1}-\mu_{2}\right) I_{2}+\beta_{2} S I_{12}, \\
I_{12}^{\prime} & =\left(b_{4}\left(1-\frac{N}{K_{4}}\right)+\alpha_{3} S+\eta_{1} I_{1}+\eta_{2} I_{2}-\mu_{3}\right) I_{12}+\left(\gamma_{1}+\gamma_{2}\right) I_{1} I_{2}, \\
R^{\prime} & =\left(b_{5}\left(1-\frac{N}{K_{5}}\right)-\mu_{4}^{\prime}\right) R+\rho_{1} I_{1}+\rho_{2} I_{2}+\rho_{3} I_{12} .
\end{aligned}
$$

Here, $S$ represents the susceptible class; $I_{1}$ and $I_{2}$ are infected classes from strain 1 and strain 2, respectively; $I_{12}$ represents coinfected class; and $R$ represents the recovered class. Finally,

$$
N=S+I_{1}+I_{2}+I_{12}+R
$$

is the total population. Here,

- $b_{i}$ is the birthrate of class $i=1,2,3,4$;

- $K_{i}$ is the carrying capacity of class $i=1,2,3,4$;

- $\rho_{i}$ is the recovery rate from each infected class $(i=1,2,3)$;

- $\beta_{i}$ is the rate of transmission of single infection from coinfected class $(i=1,2)$;

- $\gamma_{i}$ is the rate at which one infected with one strain gets infected with the other strain and moves to the coinfected class $(i=1,2)$;

- $\mu_{i}^{\prime}$ is the death rate of each class, $(i=1,2,3,4)$;

- $\alpha_{1}, \alpha_{2}, \alpha_{3}$ are rates of transmission of strain 1, strain 2, and both strains (in the case of coinfection);

- $\eta_{i}$ is the rate at which one infected from one strain gets infection from the coinfected class $(i=1,2)$ and $\mu_{i}=\rho_{i}+\mu_{i}^{\prime}$, $i=1,2,3$.

In this paper, we address a certain specialization of (1) (an SIR model with limited growth of susceptible population). More precisely, we assume that the per capita reproduction of susceptible population is limited by carrying capacity $K<\infty$ while infected and recovered individuals cannot reproduce, ie,

$$
b_{i}=0 \text { for } i \geq 2 .
$$

The corresponding SIR model reduces to the following system:

$$
\begin{aligned}
S^{\prime} & =\left(b\left(1-\frac{S}{K}\right)-\alpha_{1} I_{1}-\alpha_{2} I_{2}-\left(\beta_{1}+\beta_{2}+\alpha_{3}\right) I_{12}-\mu_{0}\right) S, \\
I_{1}^{\prime} & =\left(\alpha_{1} S-\eta_{1} I_{12}-\gamma_{1} I_{2}-\mu_{1}\right) I_{1}+\beta_{1} S I_{12}, \\
I_{2}^{\prime} & =\left(\alpha_{2} S-\eta_{2} I_{12}-\gamma_{2} I_{1}-\mu_{2}\right) I_{2}+\beta_{2} S I_{12}, \\
I_{12}^{\prime} & =\left(\alpha_{3} S+\eta_{1} I_{1}+\eta_{2} I_{2}-\mu_{3}\right) I_{12}+\left(\gamma_{1}+\gamma_{2}\right) I_{1} I_{2}, \\
R^{\prime} & =\rho_{1} I_{1}+\rho_{2} I_{2}+\rho_{3} I_{12}-\mu_{4}^{\prime} R .
\end{aligned}
$$

We consider some natural assumptions on the fundamental parameters of the system and the initial data. First note that if the reproduction rate of susceptible is less than their death rate, then the population will die out quickly. Therefore, we shall always assume that

$$
b>\mu_{0} .
$$

The system is considered under the natural initial conditions

$$
S(0)>0, \quad I_{1}(0) \geq 0, \quad I_{2}(0) \geq 0, \quad I_{12}(0) \geq 0 .
$$

Then, it follows from the standard theory (see, for example, Proposition 2.1 in the work of Haddad et $\mathrm{al}^{22}$ ) that any integral curve with (3) is staying in the nonnegative cone for all $t \geq 0$. Note also that, because the variable $R$ is not present 
in the first four equations, we may consider only the first four equations of system (1). In the work of Ghersheen et al, ${ }^{19}$ the particular case of (2) was completely treated, when the parameters $\beta_{i}$ and $\gamma_{j}$ vanish, ie,

$$
\beta_{1}=\beta_{2}=\gamma_{1}=\gamma_{2}=0 .
$$

The corresponding system has the Lotka-Volterra type, and an approach based on the linear complimentary problem was suggested. The latter allows to obtain an effective description of the transition dynamics of (2) for any admissible values of the fundamental parameters. The present model is more involved and is no longer a Lotka-Volterra system. However, thinking of (2) as a perturbation of the Lotka-Volterra case, it is reasonable to believe that some basic properties can be extended for positive small values $\beta_{i}$ and $\gamma_{j}$. Below, we shall see that this is indeed the case.

Let us introduce the reproduction/threshold numbers of the system (2) for strains 1 and 2, respectively, by

$$
\sigma_{i}:=\frac{\mu_{i}}{\alpha_{i}}, \quad 1 \leq i \leq 2
$$

Then, by change of the indices (if needed), we may assume that $\sigma_{1}<\sigma_{2}$, ie,

$$
\sigma_{1}<\sigma_{2}
$$

The letter also means that strain 1 is more aggressive than strain 2 .

Because the interaction between coinfected and susceptible classes results a single infection transmission or a simultaneous transmission of two infections,

$$
\begin{aligned}
& \beta_{1} S I_{12} \rightarrow I_{1} \\
& \beta_{2} S I_{12} \rightarrow I_{2} \\
& \alpha_{3} S I_{12} \rightarrow I_{12},
\end{aligned}
$$

it follows from Figure 1 that the corresponding reproduction/threshold number of the coinfected class is determined by

$$
\sigma_{3}=\frac{\mu_{3}}{\hat{\alpha_{3}}}=\frac{\mu_{3}}{\alpha_{3}+\beta_{1}+\beta_{2}},
$$

where

$$
\hat{\alpha_{3}}:=\alpha_{3}+\beta_{1}+\beta_{2}
$$

is the total transmission rate of infection from coinfected class to susceptible class. Note that in the Lotka-Volterra case (4) $\sigma_{3}=\frac{\mu_{3}}{\alpha_{3}}$, which is completely consistent with the notation of Ghersheen et al. ${ }^{19}$

For biological reasons, the latter total transmission rate should be less than other transmission rates comparable with the corresponding death rates. On the other hand, it is natural to assume that the death rates $\mu_{i}$ are almost the same for different classes. This makes it natural to assume the following hypotheses:

$$
\sigma_{1}<\sigma_{2}<\sigma_{3} .
$$

Finally, let us introduce an important parameter of the above system, the so-called modified carrying capacity defined by

$$
S^{* *}:=K\left(1-\frac{\mu_{0}}{b}\right)>0 .
$$

Note that $S^{* *}$ is always less than $K$, but it is proportional to $K$ whenever $b$ and $\mu_{0}$ are fixed. We study the transition dynamics of stable equilibrium states depending on the value of $K$ in Section 6 . The vector of fundamental parameters

$$
p=\left(b, K, \mu_{i}, \alpha_{j}, \eta_{k}, \gamma_{k}, \beta_{k}\right) \in R_{+}^{11}, \quad 0 \leq i \leq 3,1 \leq j \leq 3,1 \leq k \leq 2,
$$

is said to be admissible if (7) holds.

\section{3 | GENERAL FACTS ON THE SIR MODEL}

In this section, we study some basic properties for the system (2), which are essential in our analysis of stability results. We follow the approach given in the work of Ghersheen et $\mathrm{al}^{19}$ for the Lotka-Volterra case (4). 
Proposition 1. If $\left(S, I_{1}, I_{2}, I_{12}\right)(t)$ is a solution of (2) with $S(0)$ positive, then

$$
S(t) \leq \frac{1}{\frac{1}{S^{* *}}\left(1-e^{-\left(b-\mu_{0}\right) t}\right)+\frac{1}{S(0)} e^{-\left(b-\mu_{0}\right) t} .}
$$

In particular,

$$
S(t) \leq \max \left\{S^{* *}, S(0)\right\}
$$

and

$$
\limsup _{t \rightarrow \infty} S(t) \leq S^{* *}
$$

Proof. It follows from the first equation of (2) that

$$
S^{\prime}-\left(b-\mu_{0}\right) S \leq-\frac{b S^{2}}{K},
$$

which can be written as

$$
\left(S e^{-\left(b-\mu_{0}\right) t}\right)^{\prime} \leq-\frac{b}{K} e^{-\left(b-\mu_{0}\right) t} S^{2} .
$$

Dividing both sides by $\left(S^{\prime} e^{-\left(b-\mu_{0}\right) t}\right)^{2}$ and integrating from 0 to $t$ give

$$
\frac{e^{\left(b-\mu_{0}\right) t}}{S} \geq \frac{b}{K\left(b-\mu_{0}\right)}\left(e^{\left(b-\mu_{0}\right) t}-1\right)+\frac{1}{S(0)},
$$

which yields (9). Then, relations (10) and (11) follow immediately from (9).

Another important property of the general system (2) is the following.

Proposition 2 (Global estimates).

If $\left(S, I_{1}, I_{2}, I_{12}\right)(t)$ is a solution of (2) with positive initial data, then

$$
S(t)+I_{1}(t)+I_{2}(t)+I_{12}(t) \leq \max \left\{S(0)+I_{1}(0)+I_{2}(0)+I_{12}(0), \frac{b S_{m}}{\mu}\right\}
$$

for $t \geq 0$, where $\mu=\min _{0 \leq i \leq 3} \mu_{i}$ and $S_{m}=\max \left\{S^{* *}, S(0)\right\}$.

Proof. Summing up the first four equations of (2), we obtain

$$
S^{\prime}+I_{1}^{\prime}+I_{2}^{\prime}+I_{12}^{\prime} \leq\left(b-\frac{b S}{K}\right) S-\mu\left(S+I_{1}+I_{2}+I_{12}\right) .
$$

Let $y(t)=S+I_{1}+I_{2}+I_{12}$; then,

$$
y^{\prime} \leq b S_{m}-\mu y .
$$

Multiplying both sides by $e^{\mu t}$ and integrating the above equation from 0 to $t$ give

$$
y(t) \leq e^{-\mu t} y(0)+\frac{b S_{m}}{\mu}\left(1-e^{-\mu t}\right) .
$$

By (10), we have $y(t) \leq \max \left\{y(0), \frac{b S_{m}}{\mu}\right\}$, which proves our claim.

Finally, in the global stability analysis given below in Section 5, we shall need the following result established recently in the work of Ghersheen et al. ${ }^{19}$

Proposition 3. Suppose that $f(t) \in L^{p}([0, \infty)) \cap C^{1}([0, \infty))$, where $p \geq 1$, and the first $k$ higher derivatives are bounded: $f^{\prime}, \ldots, f^{(k)} \in L^{\infty}([0, \infty))$. Then,

$$
\lim _{t \rightarrow \infty} f(t)=\ldots=\lim _{t \rightarrow \infty} f^{(k-1)}(t)=0 .
$$




\section{4 | EQUILIBRIUM POINTS: THE LOCAL STABILITY ANALYSIS}

\section{1 | Basic properties}

In this section, we identify all equilibria of the system (2) in the case when

$$
\beta_{1}>0, \quad \beta_{2}>0, \quad \gamma_{1}+\gamma_{2}>0
$$

and determine their local stability properties. First, let us remark some useful balance relations, which hold for any equilibrium point $Y=\left(Y_{0}, Y_{1}, Y_{2}, Y_{3}\right)$ of (2). Because we are only interested in nonnegative equilibrium states, we always assume that

$$
Y=\left(Y_{0}, Y_{1}, Y_{2}, Y_{3}\right) \geq 0
$$

Then, we have

$$
\begin{aligned}
\left(b\left(1-\frac{Y_{0}}{K}\right)-\alpha_{1} Y_{1}-\alpha_{2} Y_{2}-\left(\beta_{1}+\beta_{2}+\alpha_{3}\right) Y_{3}-\mu_{0}\right) Y_{0} & =0 \\
\left(\alpha_{1} Y_{0}-\eta_{1} Y_{3}-\gamma_{1} Y_{2}-\mu_{1}\right) Y_{1}+\beta_{1} Y_{0} Y_{3} & =0 \\
\left(\alpha_{2} Y_{0}-\eta_{2} Y_{3}-\gamma_{2} Y_{1}-\mu_{2}\right) Y_{2}+\beta_{2} Y_{0} Y_{3} & =0 \\
\left(\alpha_{3} Y_{0}+\eta_{1} Y_{1}+\eta_{2} Y_{2}-\mu_{3}\right) Y_{3}+\left(\gamma_{1}+\gamma_{2}\right) Y_{1} Y_{2} & =0 .
\end{aligned}
$$

Denote by $G_{1}:=(0,0,0,0)$ the trivial equilibrium state. Then,

$$
Y_{0} \neq 0 \quad \text { unless } Y=G_{1} .
$$

Indeed, if $Y_{0}=0$, then we have from the second equation of (14) that $\left(\eta_{1} Y_{3}+\gamma_{1} Y_{2}+\mu_{1}\right) Y_{1}=0$. However, by the positivity assumption, $\eta_{1} Y_{3}+\gamma_{1} Y_{2}+\mu_{1} \geq \mu_{1}>0$; hence, $Y_{1}=0$. For the same reason, we have $Y_{2}=0$; thus, the last equation in (14) yields

$$
\mu_{3} Y_{3}=\left(\gamma_{1}+\gamma_{2}\right) Y_{1} Y_{2}=0
$$

hence, $Y_{3}=0$ too. This proves that $Y=G_{1}$ and proves (15).

It follows that any nontrivial equilibrium state $Y \neq G_{1}$ must satisfy

$$
b\left(1-\frac{Y_{0}}{K}\right)-\alpha_{1} Y_{1}-\alpha_{2} Y_{2}-\left(\beta_{1}+\beta_{2}+\alpha_{3}\right) Y_{3}-\mu_{0}=0,
$$

which implies by (8) and (6) the balance equation

$$
\alpha_{1} Y_{1}+\alpha_{2} Y_{2}+\hat{\alpha_{3}} Y_{3}=\frac{b}{K}\left(S^{* *}-Y_{0}\right)
$$

In addition, summing up equations in (14), we obtain

$$
\mu_{1} Y_{1}+\mu_{2} Y_{2}+\mu_{3} Y_{3}=\frac{b}{K}\left(S^{* *}-Y_{0}\right) Y_{0}
$$

Taking into account (15), the latter identities imply a priori bounds for $Y_{0}$.

Lemma 1. Let $Y \neq G_{1}$ be a nontrivial equilibrium point of (2). Then,

$$
0<Y_{0} \leq S^{* *},
$$

and the equality holds if and only if $Y_{1}=Y_{2}=Y_{3}=0$. Furthermore,

$$
\sigma_{1} \leq Y_{0} \leq \min \left\{S^{* *}, \sigma_{3}\right\}
$$

unless $Y_{0}=S^{* *}$.

Proof. Indeed, the first claim follows immediately from (16). Next, assuming that $Y_{0} \neq S^{* *}$ and dividing (17) by (16), we get $Y_{0}=\frac{\mu_{1} Y_{1}+\mu_{2} Y_{2}+\mu_{3} Y_{3}}{\alpha_{1} Y_{1}+\alpha_{2} Y_{2}+\alpha_{3} Y_{3}}$, which readily yields (19). 
Combining the above estimates, we obtain from (16) the following a priori bound on the coordinates of an arbitrary equilibrium point $Y$ distinct from $G_{2}:=\left(S^{* *}, 0,0,0\right)$ that

$$
\|Y\|_{\infty}:=\max _{0 \leq i \leq 3} Y_{i} \leq \max \left\{\frac{b-\mu_{0}}{\alpha_{1}}, \frac{b-\mu_{0}}{\alpha_{2}}, \frac{b-\mu_{0}}{\hat{\alpha}_{3}}, \sigma_{3}\right\} .
$$

\section{2 | Equilibrium points of (2)}

Note that (2) always has the trivial equilibrium state

$$
G_{1}=(0,0,0,0) .
$$

The first nontrivial equilibrium point is the disease-free equilibrium (or a healthy equilibrium), which is an equilibrium such that the disease is absent in all the patches. In the present notation, the disease-free equilibrium corresponds to $I_{1}=I_{2}=I_{12}=0$, and it follows from (2) that

$$
G_{2}=\left(S^{* *}, 0,0,0\right) .
$$

Then, Lemma 1 shows that the value of the susceptible class for the healthy equilibrium state $G_{2}$ is the largest possible among all equilibrium points.

Suppose that $Y_{3}=0$. Then, it follows from (14) that, besides $G_{2}$, there exist exactly two equilibrium points with the presence of the first or the second strains, given respectively by

$$
G_{3}=\left(\sigma_{1}, \frac{b}{K \alpha_{1}}\left(S^{* *}-\sigma_{1}\right), 0,0\right) \quad \text { when } \quad S^{* *}>\sigma_{1}
$$

and

$$
G_{4}=\left(\sigma_{2}, 0, \frac{b}{K \alpha_{2}}\left(S^{* *}-\sigma_{2}\right), 0\right) \text { when } S^{* *}>\sigma_{2} .
$$

Finally, suppose that $Y$ is a nontrivial equilibrium point such that $Y_{3} \neq 0$. Because $Y_{0} \neq 0$, the second and the third equations in (14) immediately imply that $Y_{1} \neq 0$ and $Y_{2} \neq 0$ as well. Thus, $Y_{3} \neq 0$ implies that $Y$ must have all positive coordinates. This equilibrium point is related to the coexistence of both strains with coinfection and is called the coexistence equilibrium point. We shall denote it by

$$
G_{5}=\left(S^{*}, I_{1}^{*}, I_{2}^{*}, I_{12}^{*}\right) .
$$

Note that, due to the complexity of our model, it is difficult to find the coordinates of the $G_{5}$-type equilibrium points explicitly. It is also a priori unclear how many such coexistence equilibrium points can exist. We address this issue in a forthcoming paper.

\subsection{The trivial equilibrium point $G_{1}$}

The Jacobian matrix for $G_{1}=(0,0,0,0)$ is

$$
J=\left[\begin{array}{cccc}
b-\mu_{0} & 0 & 0 & 0 \\
0 & -\mu_{1} & 0 & \\
0 & 0 & -\mu_{2} & 0 \\
0 & 0 & 0 & -\mu_{3}
\end{array}\right] .
$$

Because $b-\mu_{0}>0$, the trivial equilibrium point $G_{1}$ is always locally unstable. In fact, we have a stronger observation.

Proposition 4. If

$$
\kappa:=\limsup _{t \rightarrow \infty}\left(\alpha_{1} I_{1}+\alpha_{2} I_{2}+\hat{\alpha}_{3} I_{12}\right)<\left(b-\mu_{0}\right)
$$

then

$$
\liminf _{t \rightarrow \infty} S(t) \geq \frac{K}{b}\left(b-\mu_{0}-\kappa\right)
$$

In particular, if $I_{1}, I_{2}, I_{12} \rightarrow 0$ as $t \rightarrow \infty$, then $S(t)$ is separated from zero. 
Proof. By virtue of (23), we have for every $\kappa_{1} \in\left(\kappa,\left(b-\mu_{0}\right)\right)$ that there exist $t_{1}>0$ such that

$$
\alpha_{1} I_{1}+\alpha_{2} I_{2}+\hat{\alpha}_{3} I_{12} \leq \kappa_{1}
$$

holds for any $t \geq t_{1}$. It follows from the first equation of (2) that

$$
S^{\prime}(t)-\left(b-\mu_{0}-\kappa_{1}\right) S(t) \geq-\frac{b S(t)^{2}}{K}
$$

for $t \geq t_{1}$; hence,

$$
\left(S(t) e^{-\left(b-\mu_{0}-\kappa_{1}\right) t}\right)^{\prime} \geq-\frac{b}{K} e^{-\left(b-\mu_{0}-\kappa_{1}\right) t} S(t)^{2} .
$$

Dividing both sides by $\left(S^{\prime} e^{-\left(b-\mu_{0}-\kappa_{1}\right) t}\right)^{2}$ and integrating from $t_{1}$ to $t$ give

$$
\frac{e^{\left(b-\mu_{0}-\kappa_{1}\right)\left(t-t_{1}\right)}}{S(t)} \leq \frac{b}{K\left(b-\mu_{0}-\kappa_{1}\right)}\left(e^{\left(b-\mu_{0}-\kappa_{1}\right)\left(t-t_{1}\right)}-1\right)+\frac{1}{S\left(t_{1}\right)},
$$

which leads to

$$
S(t) \geq \frac{S\left(t_{1}\right)}{\frac{b}{K\left(b-\mu_{0}-\kappa_{1}\right)} S\left(t_{1}\right)\left(1-e^{-\left(b-\mu_{0}-\kappa_{1}\right)\left(t-t_{1}\right.}\right)+e^{-\left(b-\mu_{0}-\kappa_{1}\right)\left(t-t_{1}\right)}}, \quad \text { for } \quad t \geq t_{1},
$$

and gives

$$
\liminf _{t \rightarrow \infty} S(t) \geq \frac{K}{b}\left(b-\mu_{0}-\kappa_{1}\right) .
$$

Because $\kappa_{1}$ is an arbitrary number from $\left(\kappa, b-\mu_{0}\right)$, this implies (24).

\subsection{The disease-free equilibrium $\boldsymbol{G}_{2}$}

As we know by Lemma 1 , the disease-free equilibrium $G_{2}=\left(S^{* *}, 0,0,0\right)$ has the largest possible among all equilibrium points. The Jacobian matrix for $G_{2}=\left(S^{* *}, 0,0,0\right)$ is

$$
J=\left[\begin{array}{cccc}
-\left(b-\mu_{0}\right) & -\alpha_{1} S^{* *} & -\alpha_{2} S^{* *} & -\hat{\alpha_{3}} S^{* *} \\
0 & \alpha_{1} S^{* *}-\mu_{1} & 0 & \beta_{1} S^{* *} \\
0 & 0 & \alpha_{2} S^{* *}-\mu_{2} & \beta_{2} S^{* *} \\
0 & 0 & 0 & \alpha_{3} S^{* *}-\mu_{3}
\end{array}\right]
$$

and has all negative eigenvalues if $S^{* *} \leq \sigma_{1}$ and (7) holds. This implies the following proposition.

Proposition 5. The disease-free equilibrium point $G_{2}$ is locally stable whenever $S^{* *} \leq \sigma_{1}$ holds.

\subsection{The equilibrium point with the presence of the first strain $G_{3}$}

The local stability analysis of equilibrium points $G_{3}$ and $G_{4}$ is more involved. First note that it follows from (21) that $G_{3}$ is nonnegative if and only if

$$
I_{1}^{*}:=\frac{b}{K \alpha_{1}}\left(S^{* *}-\sigma_{1}\right) \geq 0,
$$

and, moreover, $G_{3}=G_{2}$ when $I_{1}^{*}=0$. Using (21), we find the corresponding Jacobian matrix evaluated at $G_{3}$ :

$$
J=\left[\begin{array}{cc}
A & \star \\
0 & B
\end{array}\right]=\left[\begin{array}{cccc}
-b \frac{\sigma_{1}}{K} & -\alpha_{1} \sigma_{1} & -\alpha_{2} \sigma_{1} & -\hat{\alpha}_{3} \sigma_{1} \\
\alpha_{1} I_{1}^{*} & 0 & -\gamma_{1} I_{1}^{*} & -\eta_{1} I_{1}^{*}+\beta_{1} \sigma_{1} \\
0 & 0 & -\alpha_{2}\left(\sigma_{2}-\sigma_{1}\right)-\gamma_{2} I_{1}^{*} & \beta_{2} \sigma_{1} \\
0 & 0 & \left(\gamma_{1}+\gamma_{2}\right) I_{1}^{*} & \alpha_{3} \sigma_{1}+\eta_{1} I_{1}^{*}-\mu_{3}
\end{array}\right],
$$


where we partitioned the Jacobian matrix into $2 \times 2$ blocks with

$$
A=\left[\begin{array}{cc}
-b \frac{\sigma_{1}}{K} & -\alpha_{1} \sigma_{1} \\
\alpha_{1} I_{1}^{*} & 0
\end{array}\right], \quad B=\left[\begin{array}{cc}
-\alpha_{2}\left(\sigma_{2}-\sigma_{1}\right)-\gamma_{2} I_{1}^{*} & \beta_{2} \sigma_{1} \\
\left(\gamma_{1}+\gamma_{2}\right) I_{1}^{*} & \alpha_{3} \sigma_{1}+\eta_{1} I_{1}^{*}-\mu_{3}
\end{array}\right] .
$$

It follows that the equilibrium point $G_{3}$ is stable if and only if both $A$ and $B$ are stable. We have for the first block matrix

$$
\operatorname{tr} A=-b \frac{\sigma_{1}}{K}<0, \quad \operatorname{det} A=\alpha_{1}^{2} I_{1}^{*} \sigma_{1}>0 ;
$$

therefore, $A$ is stable for any choice of parameters provided that $G_{3}$ exists and is distinct of $G_{2}$ (ie, $I_{1}^{*}>0$ ).

Next, notice that the matrix $B$ is stable if and only if its trace is negative and the determinant is positive. Because the first diagonal element in $B$ is negative by (7) and the anti-diagonal elements are positive, the positivity of the determinant implies that

$$
B_{22}=\alpha_{3} \sigma_{1}+\eta_{1} I_{1}^{*}-\mu_{3}<0 .
$$

Therefore, det $B>0$ implies that $\operatorname{tr} B=B_{11}+B_{22}<0$, ie, $B$, is stable. This shows that the block $B$ is stable if and only if $\operatorname{det} B>0$ holds. In summary, we have

Proposition 6. The equilibrium point with the presence of the first strain $G_{3}$ is locally stable if and only if $I_{1}^{*}>0$ and

$$
\operatorname{det} B=\operatorname{det}\left[\begin{array}{cc}
-\alpha_{2}\left(\sigma_{2}-\sigma_{1}\right)-\gamma_{2} I_{1}^{*} & \beta_{2} \sigma_{1} \\
\left(\gamma_{1}+\gamma_{2}\right) I_{1}^{*} & \alpha_{3} \sigma_{1}+\eta_{1} I_{1}^{*}-\mu_{3}
\end{array}\right]>0 .
$$

Let us consider the determinantal condition (26) in more detail. In the Lotka-Volterra case (4) treated in the work of Ghersheen et al, ${ }^{19}$ one has $\gamma_{i}=\beta_{j}=0$; hence, the corresponding determinant condition

$$
\operatorname{det} B_{0}=\operatorname{det}\left[\begin{array}{cc}
-\alpha_{2}\left(\sigma_{2}-\sigma_{1}\right) & 0 \\
0 & \alpha_{3} \sigma_{1}+\eta_{1} I_{1}^{*}-\mu_{3}
\end{array}\right]>0
$$

becomes equivalent to a simpler inequality (cf (25)),

$$
I_{1}^{*}=\frac{b}{K \alpha_{1}}\left(S^{* *}-\sigma_{1}\right)<\frac{\mu_{3}-\alpha_{3} \sigma_{1}}{\eta_{1}} .
$$

Coming back to the general case (13), the determinant

$$
\Delta(\lambda):=\operatorname{det}\left[\begin{array}{cc}
-\alpha_{2}\left(\sigma_{2}-\sigma_{1}\right)-\gamma_{2} \lambda & \beta_{2} \sigma_{1} \\
\left(\gamma_{1}+\gamma_{2}\right) \lambda & \alpha_{3} \sigma_{1}+\eta_{1} \lambda-\mu_{3}
\end{array}\right]
$$

is a quadratic polynomial in $\lambda$ with a negative leading coefficient. Further, by virtue of (5) and (7), we have

$$
\Delta(0)=\alpha_{2} \alpha_{3}\left(\sigma_{2}-\sigma_{1}\right)\left(\sigma_{3}-\sigma_{1}\right)>0
$$

hence, the equation $\Delta(\lambda)$ has a unique positive root. We denote it by $\Lambda$. Then, one can easily see that (26) is equivalent to the inequality

$$
0<I_{1}^{*}=\frac{b}{K \alpha_{1}}\left(S^{* *}-\sigma_{1}\right)<\Lambda,
$$

which is equivalent to

$$
\sigma_{1}<S^{* *}<\sigma_{1}+\frac{K \alpha_{1}}{b} \Lambda
$$

and under this condition, the equilibrium point $G_{3}$ is stable.

When $\Delta\left(I_{1}^{*}\right) \approx 0$ and negative, it is plausible to expect that $G_{3}$ bifurcates into an inner point of $G_{5}$-type. We shall consider this question in short in Section 6 below.

\subsection{The equilibrium point with the presence of the second strain $G_{4}$}

Similar to the above, $G_{4}$ is nonnegative if and only if

$$
I_{2}^{*}:=\frac{b}{K \alpha_{2}}\left(S^{* *}-\sigma_{2}\right) \geq 0 .
$$


Note that if $G_{4}$ is nonnegative, then $S^{* *} \geq \sigma_{2}$; hence, by virtue of (7), $G_{3}$ is nonnegative too. The Jacobian matrix evaluated at $G_{4}$ is

$$
J=\left[\begin{array}{cccc}
-b \frac{\sigma_{2}}{K} & -\alpha_{1} \sigma_{2} & -\alpha_{2} \sigma_{2} & -\hat{\alpha_{3} \sigma_{2}} \\
0 & \alpha_{1}\left(\sigma_{2}-\sigma_{1}\right)-\gamma_{1} I_{2}^{*} & 0 & \beta_{1} \sigma_{2} \\
\alpha_{2} I_{2}^{*} & -\gamma_{2} I_{2}^{*} & 0 & -\eta_{2} I_{2}^{*}+\beta_{2} \sigma_{2} \\
0 & \left(\gamma_{1}+\gamma_{2}\right) I_{2}^{*} & 0 & -\mu_{3}+\alpha_{3} \sigma_{2}+\eta_{2} I_{2}^{*}
\end{array}\right] .
$$

Note that the elementary row operation of the matrix (29) does not affect the eigenvalues of this matrix. Therefore, after an obvious rearrangement, the stability of $J$ is equivalent to that of the following matrix:

$$
\tilde{J}=\left[\begin{array}{ll}
C & \star \\
0 & D
\end{array}\right]
$$

with the diagonal $2 \times 2$-blocks

$$
C=\left[\begin{array}{cc}
-b \frac{S}{K} & -\alpha_{2} \sigma_{2} \\
\alpha_{2} I_{2}^{*} & 0
\end{array}\right], \quad D=\left[\begin{array}{cc}
\alpha_{1}\left(\sigma_{2}-\sigma_{1}\right)-\gamma_{1} I_{2}^{*} & \beta_{1} \sigma_{2} \\
\left(\gamma_{1}+\gamma_{2}\right) I_{2}^{*} & -\mu_{3}+\alpha_{3} \sigma_{2}+\eta_{2} I_{2}^{*}
\end{array}\right] .
$$

Therefore, $J$ is stable if and only if the blocks (30) are stable. The first block $C$ is stable provided $G_{4}$ is nonnegative. Thus, the stability of $G_{4}$ is equivalent to that of $D$. Similar to the previous case, we have the following proposition.

Proposition 7. The equilibrium point with the presence of the second strain $G_{4}$ is locally stable if and only if $S^{* *}>\sigma_{2}$, and

$$
\operatorname{det} D=\operatorname{det}\left[\begin{array}{cc}
\alpha_{1}\left(\sigma_{2}-\sigma_{1}\right)-\gamma_{1} I_{2}^{*} & \beta_{1} \sigma_{2} \\
\left(\gamma_{1}+\gamma_{2}\right) I_{2}^{*} & -\mu_{3}+\alpha_{3} \sigma_{2}+\eta_{2} I_{2}^{*}
\end{array}\right]>0
$$

and

$$
\operatorname{tr} D=\alpha_{1}\left(\sigma_{2}-\sigma_{1}\right)-\gamma_{1} I_{2}^{*}-\mu_{3}+\alpha_{3} \sigma_{2}+\eta_{2} I_{2}^{*}<0
$$

Remark 1 . Note that if (4) is satisfied, then $D$ is upper triangular and has an eigenvalue $\alpha_{1}\left(\sigma_{2}-\sigma_{1}\right)>0$, thus unstable. This shows that for the local stability of $G_{4}, \gamma_{1}$ must be a larger a priori lower bound. Because we consider (2) as a suitable modification of the Lotka-Volterra case (4), $G_{4}$ is an unstable point for small perturbations of the parameters $\gamma_{i}$ and $\beta_{j}$. The latter is completely consistent with the results in the work of Ghersheen et al. ${ }^{19}$

\section{5 | GLOBAL STABILITY OF EQUILIBRIUM POINTS}

\section{1 | The Lyapunov function}

Let us denote

$$
Y=\left(\begin{array}{l}
Y_{0} \\
Y_{1} \\
Y_{2} \\
Y_{3}
\end{array}\right)=\left(\begin{array}{c}
S \\
I_{1} \\
I_{2} \\
I_{12}
\end{array}\right)
$$

and rewrite our system (2) in notation

$$
\frac{d Y_{k}}{d t}=F_{k}(Y) \cdot Y_{k}+H_{k}, \quad k=0,1,2,3
$$

where we denote

$$
F(Y)=-q+A Y
$$

with

$$
F(Y)=\left(\begin{array}{l}
F_{0}(Y) \\
F_{1}(Y) \\
F_{2}(Y) \\
F_{3}(Y)
\end{array}\right), \quad q=\left(\begin{array}{c}
-b+\mu_{0} \\
\mu_{1} \\
\mu_{2} \\
\mu_{3}
\end{array}\right), \quad A=\left(\begin{array}{cccc}
-\frac{b}{K} & -\alpha_{1} & -\alpha_{2} & -\hat{\alpha}_{3} \\
\alpha_{1} & 0 & -\gamma_{2} & -\eta_{1} \\
\alpha_{2} & -\gamma_{1} & 0 & -\eta_{2} \\
\alpha_{3} & \eta_{1} & \eta_{2} & 0
\end{array}\right), \quad H=\left(\begin{array}{c}
0 \\
\beta_{1} Y_{0} Y_{3} \\
\beta_{2} Y_{0} Y_{3} \\
\left(\gamma_{1}+\gamma_{2}\right) Y_{1} Y_{2}
\end{array}\right)
$$


Then, $Y^{*}=\left(Y_{0}^{*}, Y_{1}^{*}, Y_{2}^{*}, Y_{3}^{*}\right)$ is an equilibrium point of (2) if and only if

$$
Y_{i}^{*} F_{i}\left(Y^{*}\right)=-H_{i}\left(Y^{*}\right), \quad 0 \leq i \leq 3 .
$$

We associate with $Y^{*}$ the Lyapunov function

$$
v_{Y^{*}}\left(Y_{0}, Y_{1}, Y_{2}, Y_{3}\right)=\sum_{i=0}^{3}\left(Y_{i}-Y_{i}^{*} \ln Y_{i}\right) .
$$

The derivative computations are slightly different from the Lotka-Volterra case considered previously and the resulting function contains both the $\gamma_{i}, \beta_{j}$ and the $H$-terms. More precisely, using consequently (34), (35), and (36), we obtain for the time derivative of $v_{Y^{*}}$ along any integral trajectory of (33)

$$
\begin{aligned}
\frac{d}{d t} v_{Y^{*}}:=\left(\nabla v_{Y^{*}}\right)^{T} \frac{d y}{d t}= & \sum_{i=0}^{3} \frac{Y_{i}-Y_{i}^{*}}{Y_{i}}\left(F_{i}(Y) Y_{i}+H_{i}(Y)\right) \\
= & \sum_{i=0}^{3} \frac{Y_{i}-Y_{i}^{*}}{Y_{i}}\left(\left(F_{i}(Y)-F_{i}\left(Y^{*}\right)\right) Y_{i}+F_{i}\left(Y^{*}\right) Y_{i}+H_{i}(Y)\right) \\
= & \sum_{i, j=0}^{3} A_{i j}\left(Y_{i}-Y_{i}^{*}\right)\left(Y_{j}-Y_{j}^{*}\right)+\sum_{i=0}^{3}\left(Y_{i}-Y_{i}^{*}\right) F_{i}\left(Y^{*}\right)+\sum_{i=1}^{3} \frac{Y_{i}-Y_{i}^{*}}{Y_{i}} H_{i}(Y) \\
= & -\frac{b}{K}\left(Y_{0}-Y_{0}^{*}\right)^{2}-\left(\gamma_{1}+\gamma_{2}\right)\left(Y_{1}-Y_{1}^{*}\right)\left(Y_{2}-Y_{2}^{*}\right)-\left(\beta_{1}+\beta_{2}\right)\left(Y_{0}-Y_{0}^{*}\right)\left(Y_{3}-Y_{3}^{*}\right) \\
& +\sum_{i=0}^{3} Y_{i} F_{i}\left(Y^{*}\right)+\sum_{i=1}^{3} H_{i}\left(Y^{*}\right)+\frac{Y_{i}-Y_{i}^{*}}{Y_{i}} H_{i}(Y) .
\end{aligned}
$$

Using (35), we find further that

$$
\frac{d}{d t} v_{Y^{*}}=-\frac{b}{K}\left(Y_{0}-Y_{0}^{*}\right)^{2}+\sum_{i=0}^{3} Y_{i} F_{i}\left(Y^{*}\right)+\Phi,
$$

where

$$
\Phi=\left(\gamma_{1}+\gamma_{2}\right)\left(Y_{1} Y_{2}^{*}+Y_{1}^{*} Y_{2}-\frac{Y_{3}^{*} Y_{1} Y_{2}}{Y_{3}}-Y_{1}^{*} Y_{2}^{*}\right)-Y_{0} Y_{3}\left(\beta_{1} \frac{Y_{1}^{*}}{Y_{1}}+\beta_{2} \frac{Y_{2}^{*}}{Y_{2}}\right)+\left(\beta_{1}+\beta_{2}\right)\left(Y_{3} Y_{0}^{*}+Y_{0} Y_{3}^{*}-Y_{0}^{*} Y_{3}^{*}\right) .
$$

Note that, in our notation (see (35)), all functions $H_{i}(Y)$ are nonnegative for any nonnegative $Y$; hence, it follows from (36) that, for any equilibrium point,

$$
F_{i}\left(Y^{*}\right) \leq 0
$$

Therefore, the sign of the derivative of the Lyapunov function depends on the sign of $\Phi$. Below, we apply the above computations to global stability results for the first two equilibrium points.

\section{2 | Global stability of equilibrium point $G_{2}$}

First, we consider the disease-free equilibrium point. Recall that, by Proposition 5, the point $G_{2}$ is locally stable if and only if

$$
S^{* *} \leq \sigma_{1}
$$

holds. Remarkably, the latter condition also implies the global stability. Furthermore, in the $G_{2}$ case, we are able to establish a global stability result that guarantees that the disease cannot invade and go extinct in small populations. In particular, the following result shows that a disease cannot persist in a small population. 
Proposition 8. Let (41) be satisfied. Then, the equilibrium point $G_{2}$ is globally asymptotically stable, ie,

$$
\begin{aligned}
\lim _{t \rightarrow \infty} I_{1}(t) & =\lim _{t \rightarrow \infty} I_{2}(t)=\lim _{t \rightarrow \infty} I_{12}(t)=0, \\
\lim _{t \rightarrow \infty} S(t) & =S^{* *} .
\end{aligned}
$$

Proof. We have for the Lyapunov function of $Y^{*}=G_{2}=\left(S^{* *}, 0,0,0\right)$ the following:

$$
v(t):=S-S^{* *} \ln S+I_{1}+I_{2}+I_{12} .
$$

We have $F_{0}\left(G_{2}\right)=0$ and $F_{i}\left(G_{2}\right)=\alpha_{i} S^{* *}-\mu_{i}, 1 \leq i \leq 3$, and $H_{i}\left(G_{2}\right)=0$ for $1 \leq i \leq 3$. Substituting this into (38) and (39), we obtain

$$
\begin{aligned}
v^{\prime}(t) & =-\frac{b}{K}\left(S^{* *}-S\right)^{2}-\left(\mu_{1}-\alpha_{1} S^{* *}\right) I_{1}-\left(\mu_{2}-\alpha_{2} S^{* *}\right) I_{2}-\left(\mu_{3}-\hat{\alpha_{3}} S^{* *}\right) I_{12} \\
& =-\frac{b}{K}\left(S^{* *}-S\right)^{2}-\alpha_{1}\left(\sigma_{1}-S^{* *}\right) I_{1}-\alpha_{2}\left(\sigma_{2}-S^{* *}\right) I_{2}-\hat{\alpha_{3}}\left(\sigma_{3}-S^{* *}\right) I_{12} \\
& \leq 0 .
\end{aligned}
$$

First, suppose that we have the strong inequality $S^{* *}>\sigma_{1}$. Then, $\sigma_{i}-S^{* *}$ are nonzero and strongly positive for any $i$. Integrating (43) over $[0, \infty]$ and using the fact that by Proposition 2 all $S, Y_{1}, Y_{2}, Y_{12}$ are bounded, we obtain

$$
\int_{0}^{\infty}\left(S-S^{*}\right)^{2} d \tau<\infty, \quad \text { and } \quad \int_{0}^{\infty}\left|I_{k}\right| d \tau=\int_{0}^{\infty} I_{k} d \tau<\infty \quad \text { for } I_{k}=I_{1}, I_{2}, I_{12} .
$$

Using again the boundedness of $S, Y_{1}, Y_{2}, Y_{12}$, in virtue of the system (2), their derivatives are also bounded. Applying Proposition 3 we conclude that $S$ converges to $S^{*}$ and $I_{1}, I_{2}, I_{12}$ converge to zero.

Now suppose that $S^{* *}=\sigma_{1}$. Because $\sigma_{i}-S^{* *}>0$ for $i=2,3$, the above argument implies that $S$ converges to $S^{*}$ and $I_{2}$, $I_{12}$ converge to zero. Let us show that $\lim _{t \rightarrow \infty} I_{1}=0$. Because $\lim _{t \rightarrow \infty} S(t)$ exist, then by Proposition $3, \lim _{t \rightarrow \infty} S^{\prime}(t) \rightarrow 0$. Therefore, the first equation in (2) implies by virtue of $\lim _{t \rightarrow \infty} S(t)=S^{* *} \neq 0$ that

$$
\frac{b}{K}\left(S(t)-S^{* *}\right)-\alpha_{1} I_{1}(t)-\alpha_{2} I_{2}(t)-\hat{\alpha}_{3} I_{12}(t) \rightarrow 0,
$$

which implies $\lim _{t \rightarrow \infty} I_{1}(t)=0$ and finishes the proof.

Remark 2. It is interesting to note that when $S^{* *}=\sigma_{1}$, we have no local stability because one eigenvalue is equal to zero. However, despite of this, due to a nonlinear character of the problem, a linear term gives the global asymptotic stability in this case.

\section{3 | Global stability of equilibrium point $G_{3}$}

Proposition 9. Let

$$
S^{* *}>\sigma_{1}
$$

and

$$
I_{1}^{*}=\frac{b}{K \alpha_{1}}\left(S^{* *}-\sigma_{1}\right) \leq \min \left\{\frac{\alpha_{2}\left(\sigma_{2}-\sigma_{1}\right)}{\gamma_{1}}, \frac{\hat{\alpha}_{3}\left(\sigma_{3}-\sigma_{1}\right)}{\eta_{1}}\right\} ;
$$

then, the equilibrium point $G_{3}$ is globally stable, ie,

$$
\lim _{t \rightarrow \infty} I_{2}(t)=\lim _{t \rightarrow \infty} I_{12}(t)=0, \quad \lim _{t \rightarrow \infty} S(t)=S^{*}, \quad \lim _{t \rightarrow \infty} I_{1}(t)=I_{1}^{*} .
$$

Remark 3. Note that the global stability condition (45) coincides with the local stability conditions (26) when (4) is satisfied. In the general case, (45) implies (26). Indeed, note that, in the notation of Section 4.5, we have by (45)

$$
B_{11}+B_{21}=-\alpha_{2}\left(\sigma_{2}-\sigma_{1}\right)-\gamma_{2} I_{1}^{*}+\left(\gamma_{1}+\gamma_{2}\right) I_{1}^{*}=-\alpha_{2}\left(\sigma_{2}-\sigma_{1}\right)+\gamma_{1} I_{1}^{*} \leq 0,
$$


and similarly,

$$
B_{12}+B_{22}=\beta_{2} \sigma_{1}+\alpha_{3} \sigma_{1}+\eta_{1} I_{1}^{*}-\mu_{3} \leq\left(\beta_{2}+\alpha_{3}\right) \sigma_{1}-\hat{\alpha}_{3} \sigma_{1}=-\beta_{1} \sigma_{1}<0
$$

hence, $B_{22}<-B_{12}<0$, and $B_{11}<-B_{21}<0$, which implies $B_{22} B_{11}>B_{12} B_{21}$. Therefore, det $B>0$. The latter implies by Proposition 6 that $G_{3}$ is locally stable.

Proof. In this case, $Y^{*}=G_{3}=\left(\sigma_{1}, I_{1}^{*}, 0,0\right)$, where $I_{1}^{*}:=\frac{b}{K \alpha_{1}}\left(S^{* *}-\sigma_{1}\right)$, and the corresponding Lyapunov function is given by

$$
v(t):=S-\sigma_{1} \ln S+I_{1}-I_{1}^{*} \ln I_{1}+I_{2}+I_{12} .
$$

Substituting this into (38) and (39) and using (45), we obtain

$$
v^{\prime}(t)=-\frac{b}{K}\left(\sigma_{1}-S\right)^{2}-\left(\alpha_{2}\left(\sigma_{2}-\sigma_{1}\right)-\gamma_{1} I_{1}^{*}\right) I_{2}-\left(\hat{\alpha}_{3}\left(\sigma_{3}-\sigma_{1}\right)-\eta_{1} I_{1}^{*}\right) I_{12}-\beta_{1} \frac{I_{1}^{*}}{I_{1}} S I_{12} \leq 0 .
$$

We suppose first that the strong inequality in (45) holds. Then, as above, integrating $(47)$ over $[0, \infty]$, we obtain

$$
\int_{0}^{\infty}\left(S-\sigma_{1}\right)^{2} d \tau<\infty, \quad \int_{0}^{\infty} I_{2} d \tau<\infty, \quad \int_{0}^{\infty} I_{12} d \tau<\infty .
$$

Therefore, by Proposition 3, $S$ converges to $\sigma_{1}$, and $I_{2}, I_{12}$ converge to zero. Now, we consider the convergence of $I_{1}$. Arguing as in the proof of Proposition 8, we obtain that $\lim _{t \rightarrow \infty} S(t)=\sigma_{1}$ and because the latter limit is nonzero, we find from the first equation in (2) that

$$
0=\lim _{t \rightarrow \infty}\left(\frac{b}{K}\left(S^{* *}-S(t)\right)-\alpha_{1} I_{1}(t)-\alpha_{2} I_{2}(t)-\hat{\alpha}_{3} I_{12}(t)\right)=\frac{b}{K}\left(S^{* *}-\sigma_{1}\right)-\alpha_{1} \lim _{t \rightarrow \infty} I_{1}(t) .
$$

This proves that $\lim _{t \rightarrow \infty} I_{1}=I_{1}^{*}$.

The case when the equality in (45) attains is studied similar to that in the proof of Proposition 8.

Remark 4. Finally remark, that a similar analysis in the case for $G_{4}$ shows that the corresponding Lyapunov function does not have a negative derivative because by assumption (7) one has

$$
\left(\alpha_{1}\left(\sigma_{2}-\sigma_{1}\right)+\gamma_{2} I_{2}^{*}\right)>0 .
$$

This indirectly shows that two infectious agents may not coexist together in the absence of coinfection.

\section{6 | TRANSITION DYNAMICS}

As it was already mentioned in the Introduction, in the work of Ghersheen et al,${ }^{19}$ the Lotka-Volterra version of our present model (2) corresponds to the vanishing of the transmission parameters (4).

In the work of Ghersheen et al, ${ }^{19}$ a very striking result has been established asserting that, for any $K>0$ and each admissible choice of the fundamental parameter,

$$
\hat{p}=\left(b, \mu_{0}, \mu_{1}, \mu_{2}, \mu_{3}, \alpha_{1}, \alpha_{2}, \alpha_{3}, \eta_{1}, \eta_{2}\right) \in R_{+}^{10},
$$

there exists exactly one stable equilibrium point $E=E(K, \hat{p})$, which depends continuously on the data $(K, \hat{p})$. It is natural to consider the transition dynamics of the stable equilibrium point $E(K, \hat{p})$ as a function of the carrying capacity $K$ keeping other parameters in $\hat{p}$ fixed. This transition dynamics has a clear biological meaning establishing the relationship between the infection transition rates $\hat{p}$ and the character of the corresponding equilibrium state. Furthermore, this also implies all possible scenarios how equilibrium states depend on the carrying capacity of the system. More precisely, in the work of Ghersheen et al, ${ }^{19}$ there were only three possible scenarios, each starting with the healthy equilibrium state for small values of $K$ and ending up at a certain equilibrium state when $K$ becomes sufficiently large, and it was referred as the continuity of the transition dynamics of stable equilibrium points. 
It is natural to expect that the continuity of the transition dynamics will hold true for small positive $\beta_{i}$ and $\gamma_{j}$, as a perturbation of (4). The numerical simulations support this conjecture. It is, however, unreasonable to believe that the latter property should hold for any positive data $\left(\beta_{1}, \beta_{2}, \gamma_{1}, \gamma_{2}\right)$. Below, we prove a part of our conjecture.

It is convenient to formulate our result for the relative carrying capacity $S^{* *}$ instead of $K$ (note that $S^{* *}$ differs from $K$ by a multiplicative constant only). Let us also introduce the threshold in (45). Namely, let $\sigma_{0}$ denote the solution of the following equation:

$$
\frac{b}{K \alpha_{1}}\left(\sigma_{0}-\sigma_{1}\right)=\min \left\{\frac{\alpha_{2}\left(\sigma_{2}-\sigma_{1}\right)}{\gamma_{1}}, \frac{\hat{\alpha}_{3}\left(\sigma_{3}-\sigma_{1}\right)}{\eta_{1}}\right\}
$$

Then,

$$
\sigma_{0}=\sigma_{0}(K)>\sigma_{1},
$$

and the global stability of $G_{3}$ holds whenever

$$
\sigma_{1} \leq S^{* *} \leq \sigma_{0} .
$$

Combining the latter with Proposition 8 and Proposition 9, we arrive at the following continuity of the transition dynamics.

Theorem 1. Let $\hat{p}>0$ be fixed. Then,

(i) if $S^{* *}$ is increasing and $0<S^{* *}<\sigma_{1}$, then the only possible stable equilibrium state of (2) is $G_{2}$;

(i') when $S^{* *}=\sigma_{1}, G_{2}$ coincides with $G_{3}$ and it is the only possible stable equilibrium state of (2);

(ii) there exists $\sigma_{0}>\sigma_{1}$ such that $G_{3}$ is the only possible stable equilibrium state of (2) for $\sigma_{1}<S^{* *}<\sigma_{0}$.

Proof. The claims immediately follow from the global stability of the corresponding equilibria (indeed, by virtue of the global stability there can exist at most one locally stable point!)

The case $S^{* *}>\sigma_{0}$ is more subtle, but we have at least some local information near $\hat{\sigma}=\sigma_{1}+\frac{K \alpha_{1}}{b} \Lambda$ and it follows from remark $3, \hat{\sigma}>\sigma_{0}$.

The following theorem describes the bifurcation of equilibrium point $G_{3}$ in the case when $\beta_{i}$ and $\gamma_{j}$ are small and $S^{* *}$ cross the threshold $\hat{\sigma}$.

Theorem 2. Let $\hat{p}>0$ be fixed and $\beta_{1}, \beta_{2}, \gamma_{1}$, and $\gamma_{2}$ are small; then, there exists $\epsilon>0$ such that, if $S^{*} \in(\hat{\sigma}, \hat{\sigma}-\epsilon)$, then the equilibrium point $G_{3}$ is unstable and the equilibrium point $G_{5}$ is stable and located near $G_{3}$.

Proof. We have a sketch of the proof. Let

$$
D:=\operatorname{det} B=\left(\alpha_{2}\left(\sigma_{2}-\sigma_{1}\right)+\gamma_{2} I_{1}^{*}\right)\left(\mu_{3}-\alpha_{3} \sigma_{1}-\eta_{1} I_{1}^{*}\right)-\beta_{2}\left(\gamma_{1}+\gamma_{2}\right) \sigma_{1} I_{1}^{*},
$$

where we keep the notation

$$
I_{1}^{*}=\frac{b}{K \alpha_{1}}\left(S^{* *}-\sigma_{1}\right)
$$

Let us consider the evolution of the equilibrium point $G_{3}$ for small values of the determinant $D$ under an additional natural condition that $S^{* *}>\sigma_{1}$. Certainly, the equilibrium point $G_{3}$ exists for any such admissible values. When $D>0$ is small, by Proposition $6, G_{3}$ is locally stable, and when $D=0$, it loses the local stability. It turns out that, at this moment, $G_{3}$ bifurcates into a pair of points: (a) it continues as $G_{3}$ for small negative values of $D$, and (b) it appears one more equilibrium point of type $G_{5}$ in a neighborhood of $G_{3}$. Indeed, to describe the latter, we will seek it by perturbation of $G_{3}$ in the form

$$
Y_{0}=\sigma_{1}+\xi_{0}, \quad Y_{1}=I_{1}^{*}+\xi_{1},
$$

where $\xi_{0}, \xi_{1}$ are small real parameters such that

$$
\lim _{D \rightarrow 0} \xi_{0}=\lim _{D \rightarrow 0} \xi_{1}=0
$$

and

$$
Y_{2}=\lambda Y_{3}, \quad Y_{3} \neq 0, \quad \lim _{D \rightarrow 0} Y_{3}=0
$$


Then, the third equation in (14) yields

$$
\lambda=\frac{\beta_{2} Y_{0}}{\mu_{2}+\gamma_{2} Y_{1}+\eta_{2} Y_{3}-\alpha_{2} Y_{0}} .
$$

For $\beta_{2}=0$, we have $\lambda=0$, and for $\beta_{2}>0$, we find

$$
\lambda=\lambda^{*}+o(1), \quad \lambda^{*}=\frac{\beta_{2} \sigma_{1}}{\mu_{2}+\gamma_{2} I_{1}^{*}-\alpha_{2} \sigma_{1}}
$$

is positive. Here, $o(1)$ denotes the rest term when $D \rightarrow 0$. From the fourth equation in (14), we obtain by elimination of $Y_{2}$

$$
\left(\mu_{3}-\alpha_{3} Y_{0}-\eta_{1} Y_{1}-\lambda \eta_{2} Y_{3}\right)\left(\mu_{2}+\gamma_{2} Y_{1}+\eta_{2} Y_{3}-\alpha_{2} Y_{0}\right)-\beta_{2}\left(\gamma_{1}+\gamma_{2}\right) Y_{0} Y_{1}=0 .
$$

Now, let us express $\xi_{0}$ and $\xi_{1}$ through $Y_{3}$. From the first two equations in (14) (with vanishing derivatives), we get

$$
\xi_{0}=\frac{1}{\alpha_{1}}\left(\eta_{1}+\lambda^{*} \gamma_{1}-\beta_{1} \frac{\sigma_{1}}{I_{1}^{*}}\right) Y_{3}+O\left(Y_{3}^{2}\right)
$$

and

$$
\xi_{1}=-\frac{1}{\alpha_{1}}\left(\lambda^{*} \alpha_{2}+\delta\right) Y_{3}-\frac{b \xi_{0}}{\alpha_{1} K}+O\left(Y_{3}^{2}\right)
$$

Using the notation (49) and setting

$$
A=\mu_{3}-\alpha_{3} \sigma_{1}-\eta_{1} I_{1}^{*}, \quad B=\alpha_{2}\left(\sigma_{2}-\sigma_{1}\right)+\gamma_{2} I_{1}^{*},
$$

we can write (50) as

$$
D+A\left(\gamma_{2} \xi_{1}+\eta_{2} Y_{3}-\alpha_{2} \xi_{0}\right)-B\left(\alpha_{3} \xi_{0}+\eta_{1} \xi_{1}+\lambda \eta_{2} Y_{3}\right)-\beta_{2}\left(\gamma_{1}+\gamma_{2}\right)\left(\sigma_{1} \xi_{1}+I_{1}^{*} \xi_{0}\right)+O\left(Y_{3}^{2}\right) .
$$

This yields

$$
Y_{3}=c D+O\left(D^{2}\right)
$$

where the coefficient $c$ can be easily evaluated in the case $\beta_{1}=\beta_{2}=0$ and $\gamma_{1}=\gamma_{2}=0$. Indeed, in this case $\lambda=0$,

$$
A=O(D) \text { and } \alpha_{3} \xi_{0}+\eta_{1} \xi_{1}=-\frac{b \eta_{1}^{2}}{K \alpha_{1}^{2}} \text {. }
$$

This yields

$$
B \frac{b \eta_{1}^{2}}{K \alpha_{1}^{2}} Y_{3}=-D+O\left(D^{2}\right)
$$

hence, we obtain (53) with

$$
c=-\frac{K \alpha_{1}^{2}}{B b \eta_{1}^{2}} .
$$

It follows from (53) that, for small negative $D$, the perturbation of $G_{3}$ bifurcates in an equilibrium point of type $G_{5}$ with positive coordinates. By the continuity argument, the latter property still holds true for small $\beta_{i}$ and $\gamma_{j}$.

\section{7 | DISCUSSION}

In this paper, we designed an SIR model as an extension of the model presented in the work of Ghersheen et $\mathrm{al}^{19}$ and observed the effect of density dependence population regulation on disease dynamics. The complete local and global stability analysis of two boundary equilibrium points revealed that, for small carrying capacity, the disease-free equilibrium point is always stable so a disease cannot persist in a small population, but for relatively large carrying capacity under some conditions, we have one globally stable endemic equilibrium point. The existence of an endemic equilibrium point 
guarantees the persistence of the disease with a possible future threat of any outbreak in the population. In the future, similar to the work of Ghersheen et al ${ }^{19}$ we would like to investigate the existence and uniqueness of the equilibrium point $G_{5}$. Because the equilibrium point $G_{3}$ loses its stability and bifurcates to the coexistence equilibrium point, it is worthwhile to show the existence of the coexistence equilibrium in that case to understand the complete dynamics of the disease. We would like to see the dynamical behavior of the system for significantly large $K$ and the case when $K=\infty$.

\section{ACKNOWLEDGEMENTS}

A PhD scholarship to Samia Ghersheen from the Higher Education Commission of Pakistan by SBK Women's University Quetta, Pakistan, is gratefully acknowledged.

\section{CONFLICT OF INTEREST}

On behalf of all authors, the corresponding author states that there is no conflict of interest.

\section{ORCID}

Vladimir Tkachev (D) https://orcid.org/0000-0002-8422-6140

\section{REFERENCES}

1. Griffiths EC, Pedersen AB, Fenton A, Petchey OL. The nature and consequences of coinfection in humans. J Infection. 2011;63(3):200-206.

2. Viney ME, Graham AL. Patterns and processes in parasite co-infection. In: Advances in Parasitology. Oxford, UK: Academic Press; 2013.

3. Anderson RM, May RM. Infectious Diseases of Humans: Dynamics and Control. Oxford, UK: Oxford University Press; 1992.

4. Glasser J, Meltzer M, Bruce L. Mathematical modeling and public policy: responding to health crises. Emerg Infect Dis. 2004;10:2050-2051.

5. Ferguson N, Anderson R, Gupta S. The effect of antibody-dependent enhancement on the transmission dynamics and persistence of multiple-strain pathogens. Proc Nat Acad Sci. 1999;96(2):790-794.

6. Kawaguchi I, Sasaki A, Boots M. Why are dengue virus serotypes so distantly related? Enhancement and limiting serotype similarity between dengue virus strains. Proc Royal Soc London B: Biol Sci. 2003;270(1530):2241-2247.

7. Sharp GB, Kawaoka Y, Jones DJ, et al. Coinfection of wild ducks by influenza A viruses: distribution patterns and biological significance. J Virology. 1997;71(8):6128-6135.

8. Chaturvedi AK, Katki HA, Hildesheim A, et al. Human papillomavirus infection with multiple types: Pattern of coinfection and risk of cervical disease. J Inf Diseases. 2011;203(7):910-920.

9. Mukandavire Z, Gumel AB, Garira W, Tchuenche JM. Mathematical analysis of a model for HIV-malaria co-infection. Math Biosci Eng. 2009;6:333-362.

10. Nthiiri JK, Lawi GO, Manyonge A. Mathematical model of pneumonia and HIV/AIDS co-infection in the presence of protection. Int $J$ Math Anal. 2015;9(42):2069-2085.

11. Abu-Raddad LJ, Patnaik P, Kublin JG. Dual infection with HIV and malaria fuels the spread of both diseases in sub-Saharan Africa. Science. 2006;314(5805):1603-1606.

12. Okosun KO, Makinde OD. A co-infection model of malaria and cholera diseases with optimal control. Math Biosci. 2014;258:19-32.

13. Castillo-Chavez C, Huang W, Li J. Competitive exclusion and coexistence of multiple strains in an SIS STD model. SIAM J Appl Math. 1999;59(5):1790-1811.

14. Zhang P, Sandland GJ, Feng Z, Xu D, Minchella DJ. Evolutionary implications for interactions between multiple strains of host and parasite. J Theor Biol. 2007;248(2):225-240.

15. Bichara D, Iggidr A, Sallet G. Global analysis of multi-strains SIS, SIR and MSIR epidemic models. J Appl Math Comput. 2014;44(1-2):273-292.

16. Martcheva M, Pilyugin SS. The role of coinfection in multidisease dynamics. SIAM J Appl Math. 2006;66(3):843-872.

17. Allen LJS, Langlais M, Phillips CJ. The dynamics of two viral infections in a single host population with applications to hantavirus. Math Biosci. 2003;186(2):191-217.

18. Gao D, Porco TC, Ruan S. Coinfection dynamics of two diseases in a single host population. J Math Anal Appl. 2016;442(1):171-188.

19. Ghersheen S, Kozlov V, Tkachev VG, Wennergren U. Dynamical behaviour of SIR model with coinfection: the case of finite carrying capacity. Math Meth Appl Sci. 2019;42(8).

20. Bremermann HJ, Thieme HR. A competitive exclusion principle for pathogen virulence. J Math Biol. 1989;27(2):179-190.

21. Zhou J, Hethcote HW. Population size dependent incidence in models for diseases without immunity. J Math Biol. 1994;32(8):809-834.

22. Haddad WM, Chellaboina V, Hui Q. Nonnegative and Compartmental Dynamical Systems. Princeton, NJ: Princeton University Press; 2010. 


\section{AUTHOR BIOGRAPHIES}

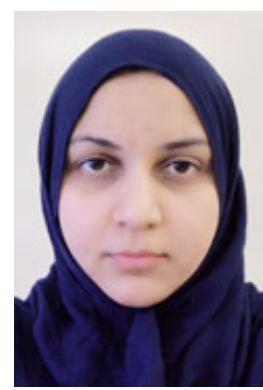

Samia Ghersheen has received his master's degree in mathematics from Pakistan. She is currently a PhD student with a scholarship in the Department of Mathematics at Linköping University, Sweden. Her research area is mathematical modeling of infectious diseases.

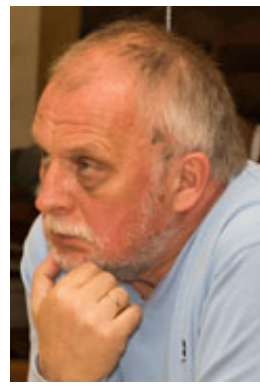

Vladimir Kozlov graduated from Leningrad University in 1976. He defended his PhD thesis in 1980 at the same University. He is a doctor of sciences in mathematics since 1990. In 1992, he moved to Linköping University, Sweden, and in 2010, he is the head of the Division of Mathematics and Applied Mathematics at the same University. Vladimir Kozlov is an author of five books and more than 160 articles. His research interests include applied mathematics (in particular, applications to theory elasticity, fluid mechanics, biology, and medicine), partial differential equations, spectral theory of operators, asymptotic methods, and inverse problems.

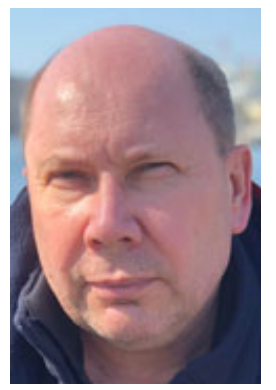

Vladimir Tkachev graduated from Volgograd University in 1985. He defended his PhD thesis in 1990 at the Sobolev Institution of Mathematics. He is a doctor of sciences in mathematics since 1998. Since 2012, Vladimir Tkachev worked as an associate professor at Linköping University, Sweden. His research interests include partial differential equations (minimal surface equation), complex analysis, and nonassociative algebras.

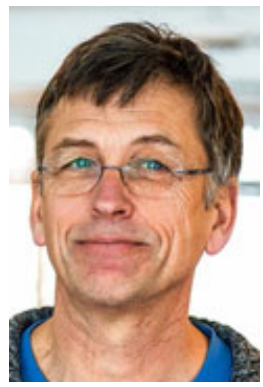

Uno Wennergren is a professor of theoretical biology at Linköping University. He took his $\mathrm{PhD}$ in 1994 by combining mathematics and biology. He did his postdoc by Peter Kareiva at the University of Washington from 1994 to 1995. Wennergren is the head of a division of 15 researchers in theoretical biology. His own research spans a wide variety of mathematical modeling of systems and process of biological relevance, from animal welfare to intricate populations dynamics of ecological systems.

How to cite this article: Ghersheen S, Kozlov V, Tkachev V, Wennergren U. Mathematical analysis of complex SIR model with coinfection and density dependence. Comp and Math Methods. 2019;1:e1042. https://doi.org/10.1002/cmm4.1042 